\title{
Characterization of the leachate produced in the closed cells of a landfill site at Alhendín (Granada, Spain)
}

\author{
A. Gálvez ${ }^{1}$, A. Ramos ${ }^{1}$, M. L. Rodríguez ${ }^{2}$ \& M. Zamorano ${ }^{1}$ \\ ${ }^{1}$ Department of Civil Engineering, University of Granada, Spain \\ ${ }^{2}$ Department of Applied Mathematics, University of Granada, Spain
}

\begin{abstract}
Landfill leachates are currently one of the main problems associated with the elimination of wastes at landfills and one of the most polluting effluents. They have a complex composition, which includes dissolved organic matter, inorganic macrocomponents, heavy metals and xenobiotic organic compounds. Leachate characterization is thus an essential factor to be considered in the design of an effective treatment system. Samples were taken of the leachate produced in the closed cells of a landfill site at Alhendín (Granada, Spain) for a period of more than one year. The analyses performed included $\mathrm{COD}, \mathrm{BOD}_{5}$, solids, ammonia, the main cations and anions, $\mathrm{pH}$, redox potential, and conductivity. The results obtained show high concentrations of different pollutants. Leachate strength followed a seasonal pattern, which can be mainly attributed to variation in rainfall and temperatures as well as evaporation rates. We found that most of the parameters analyzed showed a close correlation. The principal component analysis was applied to the data with a view to evaluating the interrelationships between parameters. It was thus possible to describe the data in terms of only four components.
\end{abstract}

Keywords: landfill leachate characterization, relationships between leachate parameters, principal component analysis.

\section{Introduction}

The high rate of waste generation in modern industrial societies has made waste management a serious urban environmental problem. Of the various waste 
management options, sanitary landfilling is still the primary method of municipal solid waste disposal in most countries. However, one of the most pervasive landfill problems that must be dealt with is leachate generation. The Landfill Directive (1999/31/CE) defines leachate as "any liquid percolating through deposited waste and emitted from or contained within a landfill".

Landfill leachate may be characterized as a water-based solution of four groups of pollutants: (i) dissolved organic matter; (ii) inorganic macro components; (iii), heavy metals; (iv) xenobiotic organic compounds [1-3]. This complex composition makes leachate one of the most polluting effluents. Consequently, leachate pollution of soil, surface water, and groundwater are frequently reported [4-7]. A proper leachate management is essential to avoid negative environmental impact. However, there is no specific technology for leachate treatment since the leachate generated at each landfill has a unique composition [8]. The reason for this is that leachate quality depends on landfill age, waste composition, landfill operation, and climate and season of the year, among other factors [1,9]. Leachate characterization is thus an essential factor in the design of an effective treatment system.

This study characterizes the leachate produced in closed cells at the Alhendín landfill site with a view to evaluating and selecting the most suitable treatment option.

\section{Materials and methods}

\subsection{Alhendín landfill site}

The leachate samples used in our study were taken from the sanitary landfill site at Alhendín (Granada). This landfill, in operation since 1999, is connected to an urban waste composting and recovery plant, and receives the waste fraction that cannot be recycled or recovered. This amounts to approximately $59 \%$ of the total waste entering the facility. The landfill is still active, but some of the zones, which have been completely filled, are now closed. Hence, active and closed cells of different ages coexist in the same landfill. The leachate produced in the landfill is collected through a drainage network that drains into several artificial ponds located in different sectors of the landfill. The leachate is recirculated back into the landfill from these ponds.

\subsection{Sampling and analysis}

The pond selected for sampling was one of the artificial ponds receiving the leachate from a closed zone of the landfill. Approximately, once a month from October 2004 to July 2006, leachate samples were collected in plastic carboys and transported to the laboratory for analysis.

As part of the leachate characterization, samples were analyzed for Chemical Oxygen Demand (COD), Biological Oxygen Demand $\left(\mathrm{BOD}_{5}\right)$, Total Suspended Solids (TSS), Volatile Suspended Solids (VSS), Fixed Suspended Solids (FSS), Total Dissolved Solids (TDS), Volatile Dissolved Solids (VDS), Fixed Dissolved Solids (FDS), Total Solids (TS), Volatile Total Solids (VTS), Fixed Total Solids 
(FTS), ammonium nitrogen $\left(\mathrm{N}-\mathrm{NH}_{4}{ }^{+}\right)$, the main anions $\left(\mathrm{F}^{-}, \mathrm{Cl}^{-}, \mathrm{Br}^{-}, \mathrm{SO}_{4}{ }^{2-}\right)$ and cations $\left(\mathrm{Na}^{+}, \mathrm{K}^{+}, \mathrm{Ca}^{2+}, \mathrm{Mg}^{2+}\right), \mathrm{pH}$, oxidation-reduction potential (Eh) and conductivity. Standard methods were used to carry out these analyses [10].

\subsection{Statistical analysis}

Data was then statistically analyzed with the software package SPSS for Windows, version 14.0 (2005). The statistical tools used for the analysis and interpretation of the data included correlation and regression analysis and principal component analysis.

\section{Results and discussion}

Our leachate samples showed high concentrations of different pollutants, in spite of being produced at closed cells of the landfill. Previous research results show that closed landfills with final covers to prevent infiltration had lower leachate production rates than active landfills [11]. Fan et al. [12] and Al-Yaqout and Hamoda [13] observed lower organic content in leachates from closed landfills as compared to leachates generated at active landfills. The pond sampled received the leachate from a landfill zone that had been active approximately from the beginning of 2003 to mid 2004. Consequently, the first samples were collected just after closing this part of the landfill, whereas the samples collected in July 2006 came from wastes that were two to three years old. Thus, this leachate was relatively young, in spite of coming from closed cells. Leachate pollutant load normally reaches maximum concentrations in the first years of landfill operation (2-3 years), and then gradually decreases over the following years [1]. Table 1 shows the results of the analysis.

COD concentrations ranged from 12158.33 to $51250.00 \mathrm{mg} / \mathrm{l}$ while $\mathrm{BOD}_{5}$ values varied from 2555.83 to $19096.00 \mathrm{mg} / \mathrm{l}$. Both COD and $\mathrm{BOD}_{5}$ concentrations were high, even though leachate samples were collected from a landfill site where most of the organic matter was previously separated from the rest of the wastes to produce compost. According to previous studies, the mechanical and biological treatment of waste before landfilling can considerably reduce the organic strength of leachates. Nevertheless, levels of nonbiodegradable organic matter in these leachates are typically as strong as, if not stronger than leachates from conventional landfills [14].

The $\mathrm{BOD}_{5} / \mathrm{COD}$ relationship also varied depending on the sample. Generally speaking, it had average values of $0.18 \pm 0.07$, which is close to the typical values for stabilized leachates [15]. This indicates a relatively low biodegradability that could be attributed to the leachate management system, consisting of leachate storage in ponds and recirculation back to the landfill, since both practices accelerate leachate stabilisation [15-17]. The $\mathrm{BOD}_{5} / \mathrm{COD}$ relationship is also known as the biodegradability factor, and its determination is essential for the selection of the most suitable treatment system [18]. For this relationship, our leachate samples showed values, ranging from 0.09 to 0.37 . This means that fixed film biological systems and physico-chemical processing are the best 
treatment options since suspended growth biological systems require values of $>0.4$ [18] for this factor.

The total solids content was also high, and ranged from 14708.43 to 58890.00 $\mathrm{mg} / \mathrm{l}$. Most of the solids were in dissolved form (97\%), given that during leachate storage, part of the suspended fraction had settled at the bottom of the ponds. The volatile content of suspended and dissolved solids was around 54\% and $37 \%$, respectively. For leachate treatment, knowledge of the values of volatile and fixed fractions of solids is essential, since only volatile fractions can be biologically degraded.

Most of the nitrogen was in the form of ammonium with concentrations between 995.10 and $2634.42 \mathrm{mg} / \mathrm{l}$. Ammonia is regarded as the principal pollutant in old leachates [15]. High ammonia concentrations can inhibit biological treatment processes [19]. Hence, its concentration is an important consideration in the design of a leachate treatment system.

Of the different ions analysed, the highest concentrations obtained were for chloride, sodium, and potassium with values of $3197.53-11212.97 \mathrm{mg} / \mathrm{l}$, 1849.24-5478.65 mg/l, and 1014.06-4541.75 mg/l, respectively.

Leachate samples were found to have a slightly alkaline $\mathrm{pH}$ with values between 7.84 and 8.12. Similar values were reported by Al-Yaqout and Hamoda [13] $(\mathrm{pH} \mathrm{7.82-8.06)}$ in old leachates. The oxidation-reduction potential was negative, with values between -59.00 and $-41.00 \mathrm{mV}$, which reflects the degree of anaerobiosis of the leachate [20]. The conductivity was high with values between 13.95 and $43.90 \mathrm{mS} / \mathrm{cm}$. Such levels indicate the presence of dissolved inorganic materials in the leachate samples [13].

As in previous studies $[13,15]$, the leachate was dark reddish-brown in colour, which can be attributed to the presence of iron [13] and humic substances [15]. The leachate also had a strong unpleasant smell. This offensive odour, also present in other landfill leachate samples [13], indicates a high content in volatile fatty acids [21].

Differences in concentration levels depended on the date of sampling. As can be observed in Table 1, leachate strength shows seasonal changes. Concentration levels are higher during July-September and lower during February-April. In all likelihood, such short-term changes in leachate quality are mainly related to the precipitation rates at the landfill [22]. Figure 1 compares average rainfall and temperature values and COD concentrations for a period of one year. As shown in Figure 1, the highest COD concentrations were obtained during the months of lower rainfall and higher temperatures, whereas the lowest COD concentrations corresponded to high precipitation rates and low temperatures. During the dry period, reduced percolation and enhanced evaporation increased leachate strength. In contrast, during the wet period, leachate was diluted with rainfall water, and thus, a higher volume of lower-strength leachate was produced. Such seasonal changes in leachate quality have also been documented by other researchers in landfill leachate samples [15, 23-25]. Evidently, this is another factor that must be considered in the design of any leachate treatment plant.

For a further analysis of the results, a bivariate analysis of Pearson correlations was applied to the different parameters with the aim of determining 


\begin{tabular}{|c|c|c|c|c|c|c|c|c|c|c|c|c|c|c|c|c|c|c|c|c|c|c|}
\hline & 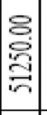 & $\begin{array}{l}8 \\
\text { 号 } \\
\text { s. }\end{array}$ & $\stackrel{8}{8}$ & 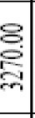 & 总 & 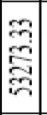 & 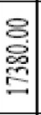 & 离 & 哭 & $\begin{array}{l}8 \\
\stackrel{8}{8} \\
8 \\
8 \\
\end{array}$ & 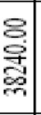 & & & & & & & & & $\left.\cdot\right|_{r} ^{\infty}$ & to & $\begin{array}{l}\curvearrowright \\
\check{子}\end{array}$ \\
\hline 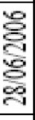 & $\mid$\begin{tabular}{l}
8 \\
$\stackrel{8}{\circ}$ \\
$\stackrel{8}{\circ}$ \\
\hdashline
\end{tabular} & $\begin{array}{l}8 \\
\stackrel{8}{8} \\
\end{array}$ & 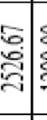 & $\stackrel{8}{8}$ & 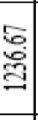 & 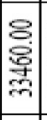 & $\begin{array}{l}\stackrel{8}{8} \\
\stackrel{8}{6} \\
\end{array}$ & 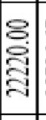 & \begin{tabular}{l}
5 \\
2 \\
2 \\
2 \\
5 \\
\hdashline
\end{tabular} & 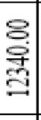 & & & & & & 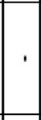 & ' & & & $\cdot$ & ๑े & ஜิ \\
\hline 옳 & $\begin{array}{l}8 \\
6 \\
2 \\
2 \\
\vdots \\
\end{array}$ & $\begin{array}{l}8 \\
\stackrel{8}{5} \\
\stackrel{్}{\circ}\end{array}$ & 8 & 胥 & 惫 & \begin{tabular}{|c|}
8 \\
8 \\
8 \\
\hdashline
\end{tabular} & $\begin{array}{l}\stackrel{8}{8} \\
\stackrel{8}{8} \\
\end{array}$ & 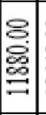 & 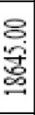 & 名 & & ' & ' & ' & & ' & ' & ' & & $\left.\cdot\right|^{\infty}$ & @㙜 & $\begin{array}{l}\mathrm{g} \\
\mathrm{g}\end{array}$ \\
\hline $\begin{array}{l}\text { '옹 } \\
\text { 옹 }\end{array}$ & $\begin{array}{l}8 \\
\stackrel{8}{8} \\
\text { \%̆ } \\
7\end{array}$ & \begin{tabular}{l}
8 \\
$\stackrel{8}{8}$ \\
\multirow{4}{*}{}
\end{tabular} & 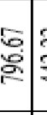 & $\begin{array}{l}\vec{z} \\
\frac{7}{8}\end{array}$ & 表 & 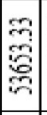 & 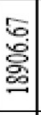 & 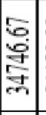 & 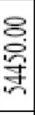 & 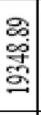 & & & & & & & & & & $\cdot \mid \infty$ & $\frac{8}{3}$ & $\begin{array}{l}8 \\
\stackrel{8}{7}\end{array}$ \\
\hline 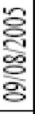 & 惡 & $\begin{array}{l}m \\
\text { o } \\
\text { 点 }\end{array}$ & 츨 & $\begin{array}{l}m \\
m \\
m \\
m\end{array}$ & 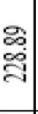 & \begin{tabular}{|c|}
8 \\
8 \\
8 \\
\hdashline \\
\hdashline
\end{tabular} & $\begin{array}{l}5 \\
\stackrel{5}{ } \\
\infty \\
\Xi\end{array}$ & 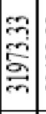 & 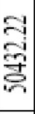 & $\begin{array}{l}\stackrel{8}{\circ} \\
\text { శై } \\
0\end{array}$ & $\begin{array}{l}\text { 今ี } \\
\text { ลิ่ }\end{array}$ & $\begin{array}{l}\Re \\
\stackrel{2}{\infty} \\
\stackrel{=}{=}\end{array}$ & 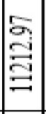 & 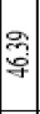 & ב & $\begin{array}{l}\infty \\
\infty \\
\approx \\
\approx\end{array}$ & $\begin{array}{l}a \\
\Xi \\
\Xi \\
\Xi\end{array}$ & 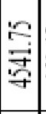 & 总 & & $8 \frac{8}{3}$ & $\begin{array}{l}8 \\
8 \\
9\end{array}$ \\
\hline $\begin{array}{l}\text { 怘 } \\
\text { 㝵 } \\
\end{array}$ & $\begin{array}{l}8 \\
\stackrel{8}{0} \\
\stackrel{2}{\mathrm{n}} \\
\end{array}$ & \begin{tabular}{l}
8 \\
0 \\
\hdashline \\
\hdashline
\end{tabular} & 文 & 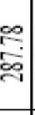 & 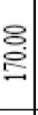 & 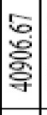 & 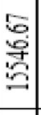 & $\mid$\begin{tabular}{l}
8 \\
0 \\
0 \\
\hdashline \\
3
\end{tabular} & 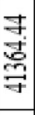 & 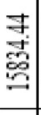 & 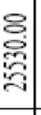 & $\stackrel{g}{\stackrel{g}{\Xi}}$ & 官 & \begin{tabular}{l}
$\approx$ \\
$\vdots$ \\
\hdashline
\end{tabular} & 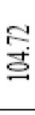 & 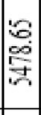 & 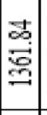 & 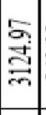 & 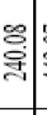 & & 8 専 & $\begin{array}{l}\text { 品 } \\
\text { 年 }\end{array}$ \\
\hline 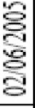 & 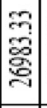 & 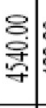 & $\begin{array}{l}\text { : } \\
\text { : } \\
\vdots \\
\end{array}$ & 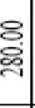 & \begin{tabular}{l}
$\infty$ \\
$\infty$ \\
$\infty$ \\
\hdashline
\end{tabular} & 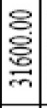 & $\begin{array}{l}\stackrel{8}{\mathscr{\Xi}} \\
\stackrel{\mathscr{E}}{=}\end{array}$ & 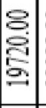 & 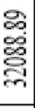 & & 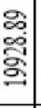 & 号 & 을 & $\stackrel{8}{8}$ & 엉 & $\begin{array}{l}\tilde{D} \\
\infty \\
\infty \\
\infty\end{array}$ & 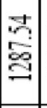 & 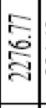 & 守 & & 용 & 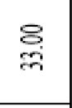 \\
\hline '气̂ें & 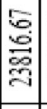 & 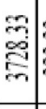 & हิ & $\begin{array}{l}\text { 寺 } \\
\stackrel{5}{a} \\
\end{array}$ & \begin{tabular}{l}
$\infty$ \\
$\infty$ \\
0 \\
\hdashline
\end{tabular} & 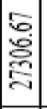 & $\begin{array}{l}8 \\
8 \\
8 \\
8\end{array}$ & $\mid \begin{array}{l}6 \\
8 \\
8 \\
6 \\
0\end{array}$ & $\begin{array}{l}\stackrel{8}{8} \\
\stackrel{0}{\circ} \\
\stackrel{0}{2}\end{array}$ & $\begin{array}{l}\text { 寻 } \\
\text { 总 } \\
\stackrel{0}{\circ}\end{array}$ & 咨 & 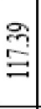 & 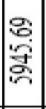 & 守 & $\underset{\sigma}{E}$ & 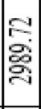 & 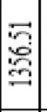 & 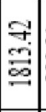 & 吾 & & $5 \frac{8}{2}$ & $\begin{array}{l}\text { శ్ } \\
\text { పิ }\end{array}$ \\
\hline 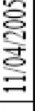 & 荵 & $\begin{array}{l}8 \\
\text { 怘 } \\
\text { 点 }\end{array}$ & 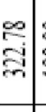 & $\stackrel{8}{8}$ & $\begin{array}{l}\infty \\
2 \\
\approx \\
=\end{array}$ & 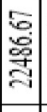 & $\frac{2}{2}$ & $\begin{array}{l}\approx \\
\stackrel{2}{2} \\
\stackrel{2}{二}\end{array}$ & $\begin{array}{l}\text { 寻 } \\
\text { 亏े } \\
\text { 今. }\end{array}$ & $\begin{array}{l}\approx \\
6 \\
6 \\
\infty\end{array}$ & 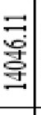 & ڤ્ & $\begin{array}{l}\stackrel{2}{8} \\
\stackrel{8}{8} \\
\frac{8}{4}\end{array}$ & $\underset{\therefore}{\approx}$ & $\therefore$ & 离 & 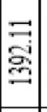 & $\begin{array}{l}\text { 焉 } \\
\text { 至 }\end{array}$ & 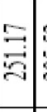 & & 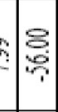 & \begin{tabular}{l}
8 \\
\hdashline \\
2
\end{tabular} \\
\hline $\begin{array}{l}\text { 今े } \\
\text { है } \\
\text { है } \\
\end{array}$ & 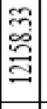 & 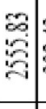 & 喜 & 号 & 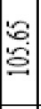 & \begin{tabular}{|}
8 \\
8 \\
8 \\
\end{tabular} & శิ & 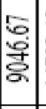 & $\begin{array}{l}\text { 甾 } \\
\text { 冚 }\end{array}$ & 㤩 & $\begin{array}{l}\vec{\sigma} \\
\text { 亏े }\end{array}$ & $\begin{array}{l}\stackrel{8}{\leftrightarrows} \\
\Leftarrow\end{array}$ & $\frac{\widehat{c}}{\mathrm{~s}}$ & 8 & 롱 & 志 & $\mid$\begin{tabular}{c}
$\mid$ \\
\hdashline \\
\hdashline \\
\hdashline
\end{tabular} & $\mid \begin{array}{l}8 \\
\\
\end{array}$ & $\begin{array}{l}\infty \\
\Xi \\
\vdots\end{array}$ & \pm & & \\
\hline 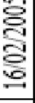 & 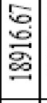 & $\begin{array}{l}8 \\
\stackrel{5}{5} \\
?\end{array}$ & $\begin{array}{l}\stackrel{8}{\Xi} \\
\dot{s}\end{array}$ & \begin{tabular}{l}
0 \\
$:$ \\
$:$ \\
\hdashline \\
\hdashline
\end{tabular} & 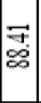 & 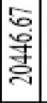 & $\begin{array}{c}\stackrel{8}{\grave{+}} \\
\stackrel{\infty}{\infty}\end{array}$ & 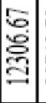 & 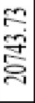 & 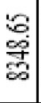 & 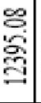 & $\frac{a}{5}$ & 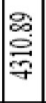 & $\begin{array}{l}8 \\
8 \\
+\end{array}$ & $\stackrel{\infty}{9}$ & 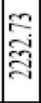 & 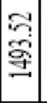 & $\mid \begin{array}{c}\overrightarrow{2} \\
\infty \\
\stackrel{\sigma}{=}\end{array}$ & $\frac{3}{3}$ & & 要 & $\begin{array}{l}\text { ळ } \\
\stackrel{9}{9}\end{array}$ \\
\hline 骨 & 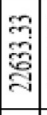 & 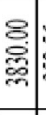 & : & 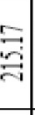 & 焉 & $\mid \begin{array}{l}5 \\
\text { 怘 } \\
\text { 离 }\end{array}$ & $\begin{array}{l}\stackrel{8}{\circ} \\
\stackrel{9}{9} \\
\end{array}$ & $\mid$\begin{tabular}{l}
6 \\
\hdashline \\
0 \\
0 \\
\hdashline
\end{tabular} & 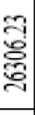 & 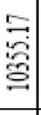 & 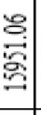 & 窇 & $\mid$\begin{tabular}{l}
$\infty$ \\
$\infty$ \\
$\infty$ \\
\hdashline \\
\hdashline
\end{tabular} & $\stackrel{\circ}{\circ}$ & $\begin{array}{l}2 \\
\hat{\delta}\end{array}$ & 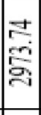 & $\mid \begin{array}{c}5 \\
\infty \\
\Xi \\
\Xi\end{array}$ & $\begin{array}{c}\text { 웜 } \\
\underset{\infty}{\infty}\end{array}$ & $\begin{array}{l}? \\
? \\
?\end{array}$ & & : & శ్ \\
\hline 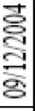 & 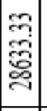 & 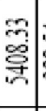 & 志 & 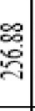 & $\stackrel{5}{-5}$ & 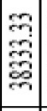 & $\begin{array}{l}8 \\
\stackrel{8}{8} \\
\stackrel{3}{=}\end{array}$ & 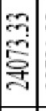 & 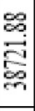 & 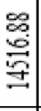 & 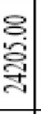 & $\begin{array}{l} \pm \\
8 \\
0 \\
0\end{array}$ & 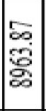 & 2 & s. & $\frac{\infty}{a}$ & 突 & $\mid$ & $\Rightarrow$ & 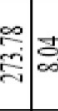 & : & $\begin{array}{l}\stackrel{0}{ } \\
\text { 出 }\end{array}$ \\
\hline 壳 & 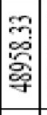 & $\begin{array}{l}8 \\
\stackrel{8}{\circ} \\
\end{array}$ & 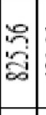 & $\begin{array}{l}\text { 三 } \\
\text { 学 }\end{array}$ & 表 & 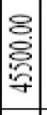 & 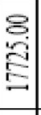 & 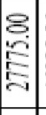 & 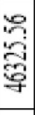 & 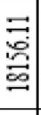 & 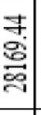 & $\begin{array}{l}F \\
\nexists 7\end{array}$ & 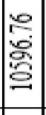 & $\stackrel{8}{\Xi}$ & $\begin{array}{l}\text { : } \\
\text { ڤै }\end{array}$ & 涼 & \begin{tabular}{l} 
고 \\
$\stackrel{8}{\Xi}$ \\
\hdashline
\end{tabular} & 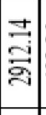 & 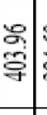 & $\begin{array}{l}8 \\
\text { 离 }\end{array}$ & $\begin{array}{l}3 \\
\vdots \\
\end{array}$ & $\begin{array}{l}8 \\
\text { \& } \\
\text { p. }\end{array}$ \\
\hline 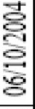 & 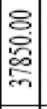 & $\begin{array}{l}8 \\
\vdots \\
5\end{array}$ & \begin{tabular}{|}
8 \\
0 \\
0 \\
0
\end{tabular} & 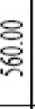 & $\begin{array}{l}\stackrel{8}{8} \\
\stackrel{8}{8} \\
\end{array}$ & 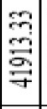 & 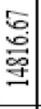 & 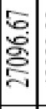 & है & 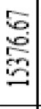 & $\begin{array}{l}\text { 혀 } \\
\text { 音 } \\
\text { 今a }\end{array}$ & 突 & 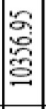 & $\stackrel{\Xi}{\Xi}$ & $\overrightarrow{8}$ & 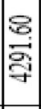 & $\begin{array}{l}2 \\
8 \\
8 \\
8\end{array}$ & $\mid$\begin{tabular}{l}
$\mathscr{2}$ \\
8 \\
8 \\
\hdashline \\
\hdashline
\end{tabular} & $\begin{array}{l}\mathrm{a} \\
\mathrm{a} \\
\mathrm{n}\end{array}$ & 골 & : & 骂 \\
\hline 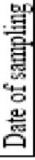 & 哥 & $\begin{array}{l}\text { 현 } \\
\text { 官 } \\
\text { q. }\end{array}$ & 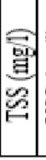 & 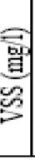 & 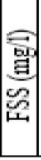 & 嬊 & 或 & 畐 & $\begin{array}{l}\text { 晋 } \\
\frac{2}{2}\end{array}$ & 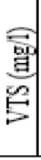 & $\begin{array}{l}\text { 䕊 } \\
\text { 点 }\end{array}$ & 볍 & 高 & 皇 & 当 & $\frac{\text { 兽 }}{\text { 运 }}$ & 畐 & 晋 & 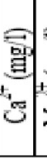 & 照 & 亭 & 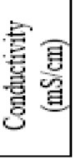 \\
\hline
\end{tabular}




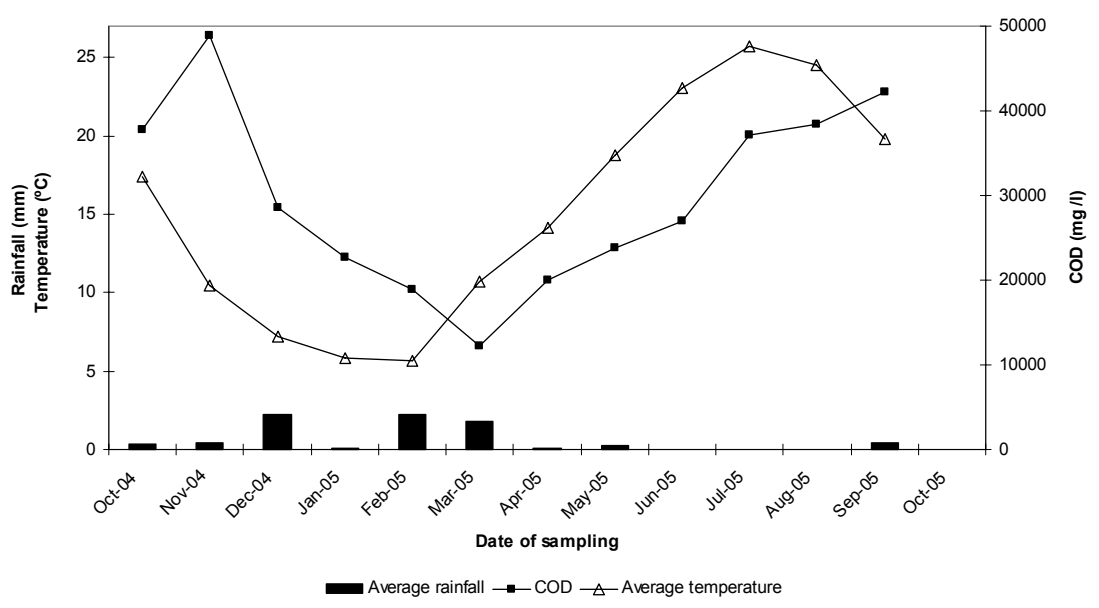

Figure 1: Relationship between average rainfall and temperature and COD concentrations.

the possible relationship between them. We found a good correlation between many of the parameters analysed. The best correlations (significant at the 0.1 level) with high Pearson correlation coefficients were obtained between the parameters marked by a dot in Table 2 .

Ammonium nitrogen only showed significant correlations at the 0.05 level with parameters $\mathrm{BOD}_{5}$ and $\mathrm{F}^{-}$. Parameters $\mathrm{Br}^{-}, \mathrm{SO}_{4}{ }^{2-}$ and $\mathrm{Ca}^{2+}$ were not correlated with any of the parameters studied. Other authors have also used this technique to analyze landfill leachate data $[12,15]$. Some of their correlations agreed with our results for Alhendín leachate samples, while others did not. This divergence can be attributed to differences in leachate composition.

A lineal regression was also applied to the pair of parameters that showed the best correlations. Some parameters showed a high level of regression with regression coefficients, $\mathrm{R}^{2}>0.9$, such as total suspended solids and volatile suspended solids with a regression coefficient of 0.9995 .

Finally, principal component analysis was applied to the leachate data in order to evaluate possible correlations between various parameters simultaneously $[8,26]$. For the analysis, 23 parameters were initially considered (COD, BOD 5 , TSS, VSS, FSS, TDS, VDS, FDS, TS, VTS, FTS, F', Cl- ${ }^{-} \mathrm{Br}^{-}$, $\mathrm{SO}_{4}{ }^{2-}, \mathrm{Na}^{+}, \mathrm{N}_{-} \mathrm{NH}_{4}{ }^{+}, \mathrm{K}^{+}, \mathrm{Ca}^{2+}, \mathrm{Mg}^{2+}, \mathrm{pH}, \mathrm{Eh}$, and conductivity). However, some of them had to be eliminated from the analysis, and the rest divided into two groups in order to meet the requirements for a valid analysis. However, a broader sampling would have allowed us to carry out a principal component analysis of all the parameters simultaneously. The first group included ten parameters $\left(\mathrm{COD}, \mathrm{BOD}_{5}, \mathrm{VDS}, \mathrm{VTS}, \mathrm{FTS}, \mathrm{F}^{-}, \mathrm{Cl}^{-}, \mathrm{Na}^{+}, \mathrm{K}^{+}, \mathrm{Ca}^{2+}, \mathrm{Mg}^{2+}\right)$ and the second group, eight parameters (FSS, VSS, TDS, FDS, TS, Eh, pH, conductivity). Five parameters (TSS, N-NH${ }_{4}^{+}, \mathrm{Ca}^{2+}, \mathrm{Br}^{-}, \mathrm{SO}_{4}{ }^{2-}$ ) were excluded from the analysis 


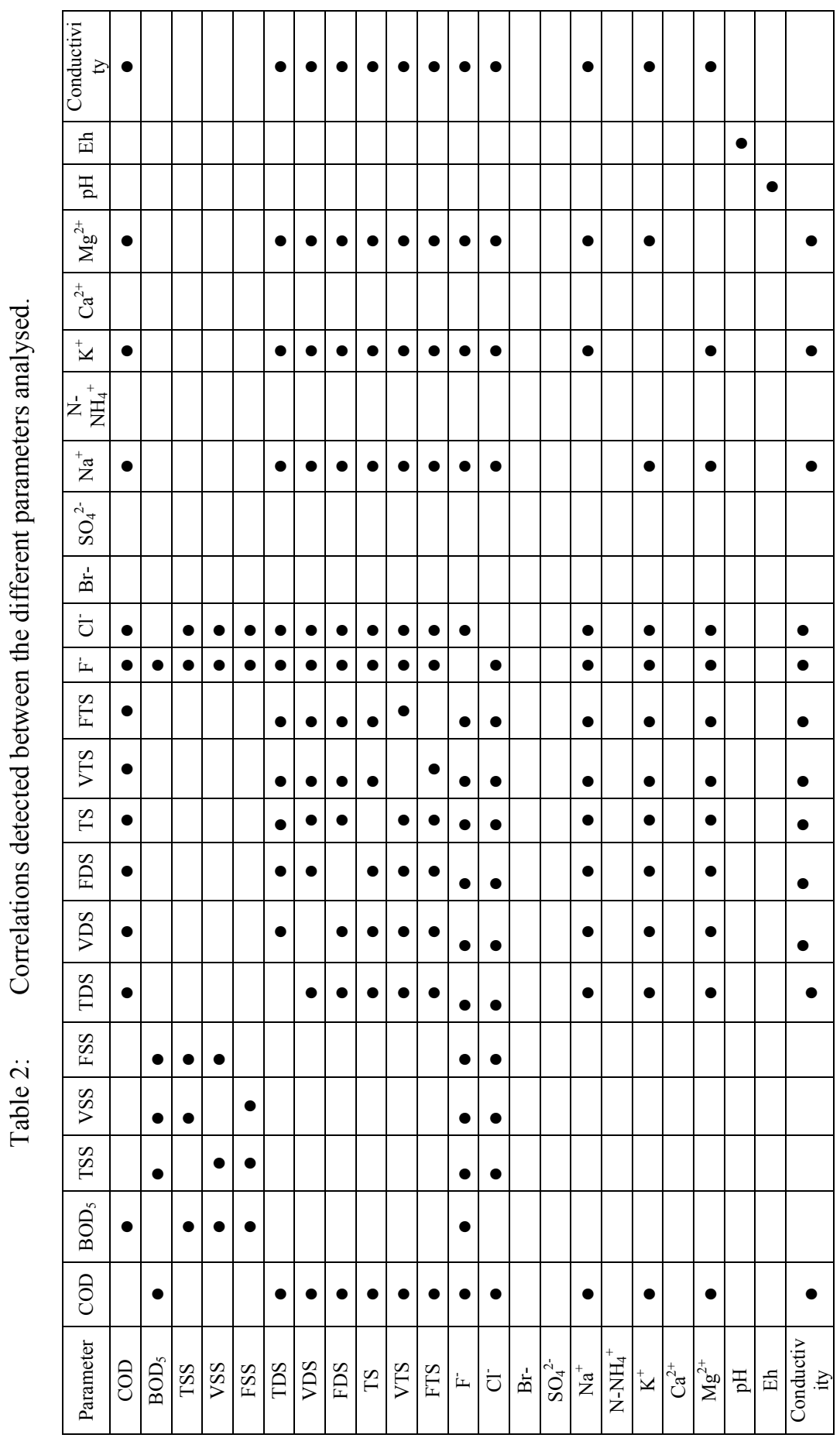


After the analysis, the parameters were subsequently reduced to four components. The first component included the parameters, $\mathrm{K}^{+}, \mathrm{Mg}^{2+}, \mathrm{FTS}, \mathrm{VDS}$, VTS, $\mathrm{Na}^{+}$and $\mathrm{Cl}^{-}$. The second component was made up of $\mathrm{BOD}_{5}$, with $\mathrm{COD}$ and $\mathrm{F}^{-}$between the first and second component. The third component included the parameters, TDS, TS, FDS and conductivity. Finally, the fourth component was made up of the parameters, $\mathrm{pH}$, FSS, Eh and VSS, with pH negatively correlated with the rest of the parameters of this fourth component.

These relationships may provide a useful means for estimating leachate strength and characteristics on site, since some of the chemical parameters can be obtained more simply than others [12]. The determination of a set of easily analyzed parameters such as solids, $\mathrm{pH}$ and conductivity may facilitate the prediction of other important pollution parameters. The information thus obtained is crucial to both the design and effective management of the leachate treatment plant [15]. It should be designed taking into consideration the maximum concentrations of pollutants and should be sufficiently flexible to adapt to those seasonal changes on leachate quality and quantity observed.

\section{Conclusions}

Leachate samples collected from a pond that received the leachate produced in closed cells at the Alhendín landfill site showed high concentrations of different pollutants. Seasonal changes in leachate composition were also observed. Most of the parameters analysed were highly correlated, and some of them showed good regression coefficients. Principal component analysis was used to reduce leachate data to four components. The results obtained in this study provide valuable information for the selection of the best leachate treatment option.

\section{Acknowledgements}

We are grateful to the company Fomento de Construcciones y Contratas S.A. (FCC) for allowing the collection of leachate samples from the Alhendín landfill site. Funding for this research study was received from the Spanish Ministry of Science and Technology (FIT-050000-2002-7).

\section{References}

[1] Christensen, T.H., Cossu, R. \& Stegmann, R., (eds). Landfilling of Waste: Leachate, Elsevier Applied Science: London and New York, 1994.

[2] Christensen, T.H., Kjeldsen, P., Bjerg, P.L., Jensen, D.L., Christensen, J.B.; Baun, A., Albrechtsen, H.-J. \& Heron, G., Biochemistry of landfill leachates plumes. Review. Applied Geochemistry 16, pp. 659-718, 2001.

[3] Kjeldsen, P., Barlaz, M.A., Rooker, A.P., Baun, A., Ledin, A. \& Christensen, T.H., Present and Long-Term Composition of MSW Landfill Leachate: A Review. Critical Reviews in Environmental Science and Technology 32(4), pp. 297-336, 2002. 
[4] Ahel, M., Mikac, N., Cosovic. B., Prohic, E. \& Soukup, V., The impact of contamination from a municipal solid waste landfill (Zagreb, Croatia) on underlying soil. Water Science and Technology 37(8), pp. 203-210, 1998.

[5] Mwiganga, M. \& Kansiime, F., The impact of Mpererwe landfill in Kampala-Uganda, on the surrounding environment. Physics and Chemistry of the Earth 30, pp. 744-750, 2005.

[6] Haq, I., Environmental impact assessment study: leaching of chemical contaminants from a municipal dump site Hastsal, Delhi (capital of India). Intern. J. Environ. Studies 60(4), pp. 363-377, 2003.

[7] Chofqi, A., Younsi, A., Lhadi, E.K., Mania, J., Mudry, J. \& Veron, A., Environmental impact of an urban landfill on a coastal aquifer (El Jadida, Morocco). Journal of African Earth Sciences 39, pp. 509-516, 2004.

[8] Kylefors, K., Evaluation of leachate composition by multivariate data analysis (MVDA). Journal of Environmental Management 68, pp. 367376, 2003.

[9] Qasim, S. R. \& Chiang, W., Sanitary landfill leachate. Generation, control and treatment, Technomic Publishing Company: Florida, USA, Inc., 1994.

[10] APHA, AWWA \& WPCF, Standard methods for the examination of water and wastewater, 17th edition; American Public Health Association: Washington, DC, 1989.

[11] Pavelka, C., Loehr, R.C. \& Haikola, B., Hazardous waste landfill leachate characteristics. Waste Management, 13(8), pp. 573-580, 1993.

[12] Fan, H.-J., Shu, H.-Y., Yang, H.-S. \& Chen, W.-C., Characteristics of landfill leachates in central Taiwan. Science of the Total Environment, 361(1-3), pp. 25-37, 2006.

[13] Al-Yaqout, A.F. \& Hamoda, M.F., Evaluation of landfill leachate in arid climate. A case study. Environment International, 29, pp. 593-600, 2003.

[14] Robinson, H.D., Knox, K., Bone, B.D. \& Picken, A., Leachate quality from landfilled MBT waste. Waste Management, 25, pp. 383-391, 2005.

[15] Tatsi, A.A. \& Zouboulis, A.I., A field investigation of the quantity and quality of leachate from a municipal solid waste landfill in a Mediterranean climate (Thessaloniki, Greece). Advances in Environmental Research, 6, pp. 207-219, 2002.

[16] Reinhart, D. R., Full-Scale experiences with leachate recirculating landfills: case studies. Waste Management and Research, 14, pp. 347-365, 1996.

[17] Diamadopoulos, E., Characterization and treatment of recirculationstabilized leachate. Water Research, 28(12), pp. 2439-2445, 1994.

[18] Gómez, M.A. \& Hontoria, E., Técnicas Analíticas en el control de la ingeniería ambiental. Granada, 2001

[19] Li, X.Z. \& Zhao, X.L., Efficiency of biological treatment affected by high strength of ammonium-nitrogen in landfill leachate and chemical precipitation of ammonium-nitrogen as pre-treatment. Chemosphere, 44, pp. 37-43, 2001.

[20] Lo, I. M. C., Characteristics and treatment of leachates from domestic landfills. Environment International, 22(4), pp. 433-442, 1996. 
[21] Inanc, B., Calli, B. \& Saatci, A., Characterization and anaerobic treatment of the sanitary landfill leachate in Istanbul. Water Science and Technology, 41(3), pp. 223-230, 2000.

[22] Statom, R. A., Thyne, G. D. \& McCray, J. E., Temporal changes in leachate chemistry of a municipal solid waste landfill cell in Florida, USA. Environmental Geology, 45, pp. 982-991, 2004.

[23] Chu, L.M., Cheung, K.C. \& Wong, M.H., Variations in the chemical properties of landfill leachate. Environ. Manage., 18(1), pp. 105-117, 1994.

[24] Akesson, M. \& Nilsson, P., Seasonal changes of leachate production and quality from test cells. Journal of Environmental Engineering, 123(9), pp. 892-900, 1997.

[25] Vadillo, I., Andreo, B., García, A. \& Bosch, C., Chemical composition of landfill leachate in a karst area with a Mediterranean climate (Marbella, southern Spain). Environmental Geology, 37(4), pp. 326-332, 1999.

[26] Durmusoglu, E. \& Yilmaz, C., Evaluation and temporal variation of raw and pre-treated leachate quality from an active solid waste landfill. Water, Air and Soil Pollution, 171, pp. 359-382, 2006. 